\title{
Presentación
}

\section{Narrativas sobre los Procesos de Inclusión y Exclusión de las Disidencias Culturales, Étnicas, Políticas, de Edad, Sexuales y de Género en la Educación Escolar en Chile y Brasil}

\author{
Narratives on the Processes of Inclusion and Exclusion of \\ Cultural, Ethnic, Political, Age, Sexual and Gender Dissidences \\ in School Education in Chile and Brazil
}

\author{
Leonardo Lemos de Souza ${ }^{1 *}$ \\ Raquel Gonçalves Salgado ${ }^{2}$ \\ ${ }^{1}$ Universidad Estatal Paulista Julio de Mesquita Filho, Brasil \\ ${ }^{2}$ Universidad Federal de Rondonópolis, Brasil
}

Establecer y revitalizar el debate democrático, tanto en el campo macropolítico como en el campo de la educación, implica una mirada cuidadosa a las disidencias desde la apertura a la reflexión crítica y la construcción de prácticas y políticas marcadas por la diversidad de pensamientos y experiencias. No hay posibilidades de pensar y vivir prácticas de inclusión cuando uno asume la disonancia como ruido que debe ser combatido o cuando el otro, en razón de las diferencias que presenta, es percibido como una amenaza y lo que debe ser aniquilado. La comprensión y el ejercicio de la inclusión, en todos sus matices culturales, étnicos, políticos, de edad, sexuales y de géneros- nos pone ante la cuestión de la alteridad, al necesario reconocimiento del otro como alguien que nos desafía y nos pone en frente nuestra precariedad, la imposibilidad de nuestra autosuficiencia y la apertura al aprendizaje que permite la resignificación de la vida subjetiva y de la cultura.

En este sentido, al abrir los artículos que presentamos en la sección monográfica de este número, conviene preguntarnos: ¿cómo tratamos, como educadores e investigadores, las experiencias, culturas, prácticas sociales, narrativas, formas de ser y de vivir del otro, niños y jóvenes, en sus disidencias culturales, étnicas, políticas, de edad, sexuales y de género no-hegemónicas en la educación escolar en países latinoamericanos, como Chile y Brasil? ¿Qué voces de niños y jóvenes escuchamos -o buscamos escuchar- cuando asumimos la tarea de educarlos/educarlas o incluso cuando estamos inmersos en procesos de investigación con ellos/ellas? ¿Cómo las políticas de austeridad neoliberal presentes en estos dos países producen exclusiones, desigualdades y violencias en la forma de racismo, sexismo, homo y transfobia en la escuela?

Son los desafíos provocados por estas preguntas, los que nos presentan desde diferentes perspectivas y problemas, los artículos recogidos en este monográfico.

*Contacto: leonardo.lemos@unesp.br 
El artículo "Diferencias morales y fronteras sociales. Los límites de la inclusión en la educación sexual de los colegios católicos de Santiago”, de Pablo Astudillo Lizama, expone los resultados de una investigación realizada en 16 colegios católicos de élite en Santiago de Chile. Los datos fueron recogidos a través de entrevistas realizadas a psicólogos y orientadores implicados en la educación sexual, así como a través grupos focales y observaciones etnográficas en el contexto de actividades formativas con adolescentes que estudian en dichos colegios. Se propone discutir el concepto de inclusión predominante en estas instituciones educativas, fuertemente pautado por posturas morales alineadas con las posiciones socioeconómicas, lo que invisibiliza las experiencias de alteridad de los jóvenes relacionados con las diversidades sexuales y de género, cuyas disposiciones provienen de las formas en que encarnan los valores impuestos en la cultura.

Las representaciones sociales de profesores y estudiantes respecto de la inclusión de personas LGBTI en una escuela secundaria católica y otra laica de Santiago, es el foco del estudio de caso que presenta el artículo de Evelyn Toro Villagra, Pablo Moya Rojas y Rolando Poblete Melis. Aunque existe preocupación por parte de las políticas educativas chilenas actuales con la inclusión del tema de la diversidad sexual, los autores analizan que aún queda mucho por hacer para romper con las ideas heteronormativas que atraviesan el debate en torno a la sexualidad en el campo de la educación en ese país.

El artículo "Infancia, género y sexualidades: una investigación-intervención con profesoras de educación infantil”, de Eduardo Benedito Cólis y Leonardo Lemos de Souza, analiza los significados producidos por educadoras del nivel inicial de una institución pública en Brasil, respecto del género, sexualidad e infancia y cómo estos repercuten en sus prácticas educativas. A través de un proceso de investigación - intervención, los autores discuten, en entrevistas y talleres realizados con las participantes, lo que piensan sobre las experiencias de los niños marcados por el género y la sexualidad y, como se enfrentan a dispositivos visibles que producen normas para la infancia que son activadas desde la escuela.

El cuarto artículo de Matheus Estevão Ferreira da Silva, "Relevancia de los temas de género y sexualidades para la educación escolar de los niños: ¿qué piensa el pedagogo?”, analiza los resultados de una investigación realizada con estudiantes universitarios/as de los años iniciales y finales del curso de Pedagogía de una universidad pública de San Pablo, Brasil, con el objetivo de analizar sus concepciones sobre la importancia de los temas de género y sexualidad en la formación y la práctica profesional. El autor destaca en sus análisis, las posiciones de valoración de las y los estudiantes sobre estos temas, como las principales referencias que adoptan para reconocer su importancia en la educación escolar.

En el debate por una educación inclusiva en el contexto chileno, los trabajos presentados en la sección libre de este número, agregan temas que, desde diferentes perspectivas y propuestas, problematizan los procesos de inclusión en la educación escolar.

En primer lugar, en el artículo "Elementos para comprender la concentración de estudiantes extranjeros en escuelas chilenas”, las autoras Claudia Córdoba Calquin, Carolina Altamirano González y Karina Rojas Patuelli, aportan al debate actual relacionado con el flujo migratorio recibido por Chile en las últimas décadas y como la concentración de estudiantes inmigrantes en determinadas escuelas representaría una nueva forma de segregación escolar. Este trabajo presenta un estudio etnográfico desarrollado en escuelas municipales que cuentan con estudiantes extranjeros en distinta proporción. A través de entrevistas realizadas a los padres y madres de los estudiantes, 
encontraron elementos discursivos que pueden explicar la tendencia a la concentración de estudiantes extranjeros en algunas escuelas, entre ellos las estrategias que utilizan las propias familias para proteger a sus hijos/as de los prejuicios y prácticas de exclusión dada su condición de migrante, y que cuestionan al mismo tiempo los procesos de selección, admisión y requisitos escolares para la inclusión de sus hijos.

Por su parte, Ignacio Figueroa-Céspedes y Cristopher Yáñez-Urbina en su trabajo sobre "Voces y culturas estudiantiles en la escuela: Una reflexión teórico-metodológica desde procesos de autoexploración de barreras a la inclusión educativa”, los autores desarrollan un análisis reflexivo como resultado de procesos de investigación acción implementados con diferentes colectivos estudiantiles en escuelas municipales de Santiago de Chile. Lo relevante de esta experiencia es que recoge la voz de los estudiantes para conocer los mecanismos de autoexploración que desarrollan las instituciones sobre las diferentes formas de existencia y de estar en la escuela. En este contexto, se relevan procesos de invisibilización de las diferencias en la cotidianidad escolar, generando, al mismo tiempo, un espacio dialogante de negociaciones de significados sobre estas diferencias, destacando el monoculturalismo, el adultocentrismo y la homogeneización de los aprendizajes en la escuela.

Juan Pablo Espinoza y Miguel de Aguilera en "Nacionalismo y narrativas nacionales en el libro de texto de enseñanza secundaria de historia de Chile", desarrollan un análisis crítico sobre el tratamiento de las ideologías del nacionalismo en textos escolares. Las ideologías nacionalistas pueden producir discursos de exclusión de sujetos y realidades, borrando de la historia las disidencias culturales, sexuales, sociales y políticas. Los análisis realizados por los autores en textos de historia de educación secundaria durante los gobiernos de Eduardo Frei y Salvador Allende (1964-1973), muestran elementos para afirmar el mantenimiento de un concepto ampliado de nación y no excluyente, que opera en el sentido contrario a una colisión entre un Nosotros y Otros (interno y externo a la nación), pero integrando las disidencias. La educación escolar vinculada a la negativa de esta colisión rompe con los silencios que crearían fisuras en el proceso civilizador de una nación que se compone de diferencias y diversidades.

En el artículo titulado, "Comprendiendo el diseño universal desde el paradigma de apoyos: DUA como un sistema de apoyos para el aprendizaje”, Victoria Sanchéz-Goméz y Mauricio López tejen consideraciones a partir de un análisis y revisión del Diseño Universal de Aprendizaje (DUA) y desarrollan una propuesta teórica orientada a favorecer una planificación curricular con impacto efectivo en el aprendizaje de los estudiantes con y sin discapacidad. Lo hacen desde la propuesta de vincular el DUA al paradigma de apoyos al aprendizaje, proporcionando una mayor proximidad a las necesidades educativas de los estudiantes con discapacidad en contextos inclusivos.

Finalmente cerramos este número, con el artículo "Representaciones sociales sobre experiencias educativas de jóvenes que presentan Trastorno del Espectro Autista (TEA) en Chile", de Camila Andrea Toledo Manríquez y Oscar Fernando Basulto Gallegos. Esta investigación se desarrolla en la ciudad de Concepción e indaga en las experiencias educativas de cinco estudiantes con TEA. A partir de análisis de las historias de vida de estos jóvenes, construidas desde las narrativas de sus madres, emergen representaciones sociales ligadas a la discriminación, la exclusión, temores en relación al futuro, entre otras. Las madres reportan dificultades en el proceso educativo, insuficiente preparación y disposición de los profesores para abordar las necesidades de sus hijos/as. El artículo, 
asimismo, aporta reflexiones críticas respecto del debate sobre los vacíos y negligencia de la legislación desde determinada edad y la producción de representaciones sobre la educación escolar como no inclusiva y discriminatoria para los estudiantes con TEA.

Esperamos que los lectores se sumerjan en las diversas perspectivas que ofrecen los trabajos que presentamos en esta nueva edición de la Revista Latinoamericana de Educación Inclusiva. Los análisis e investigaciones llevados a cabo dan cuenta de los desafíos que involucran los procesos de construcción de una educación escolar inclusiva efectiva en países como Chile y Brasil. Lo que los estudios revelan es que estos países todavía están en una lucha permanente a fin de generar espacios democráticos y reducir las desigualdades, especialmente en la lucha contra las narrativas sustentadas en la normalización, patologización y discriminación de las diferencias. La invisibilidad de las diferentes formas de expresión cultural, política, social, de aprendizaje, de edad, sexual y de género se plantean en los textos como barreras a superar. Al mismo tiempo, la afirmación de estas existencias se presenta como resistencia en la cotidianeidad escolar, produciendo fisuras y desviaciones develadas por la investigación y las prácticas pedagógicas/educativas comprometidas con el desarrollo de una escuela democrática e inclusiva.

\section{Breve CV de los autores}

\section{Leonardo Lemos de Souza}

Doctor en Educación por la Universidade Estadual de Campinas. Postdoctorado en el Departamento de Psicología Básica de la Universitat de Barcelona. Profesor Asociado del Departamento de Psicología Social y del Programa de Posgrado en Psicología de la Universidade Estadual Paulista Julio de Mesquita Filho- Unesp. Vinculado al Grupo de Investigación de Psicología, Colectivos y Culturas Queer. ORCID ID: https://orcid.org/0000-0002-3331-1847 - Email: leonardo.lemos@unesp.br

\section{Raquel Gonçalves Salgado}

Doctora en Psicología por la Pontifícia Universidade Católica do Rio de Janeiro (PUCRio). Profesora Asociada de la Universidade Federal de Rondonópolis (UFR). Vinculada al Programa de Posgrado en Educación de la UFR y al Grupo de Investigación Infância, Juventude e Cultura Contemporânea. ORCID ID: https://orcid.org/0000-0002-87303025. Email: ramidan@terra.com.br 